\title{
THE VIBRATION OF NANOBEAM RESTING ON ELASTIC FOUNDATION USING MODIFIED COUPLE STRESS THEORY
}

\author{
Necla TOGUN, Süleyman M. BAĞDATLI
}

\begin{abstract}
In this paper, the vibration of nanobeams resting on the Winkler foundation is proposed using the modified couple stress theory. Hamilton's principle is utilized to construct the governing equations. The size effect of the nanobeam cannot be captured by using classical Euler-Bernoulli beam theory, but the modified couple stress theory model can capture it because it includes material length scale parameter that a newly developed model has. Once the material length scale parameter is assumed to be zero, the classical Euler-Bernoulli beam theory equation is obtained. Multiple scale method is employed to obtain the result. Simply supported boundary condition is used to study natural frequencies. The influence of material length scale parameter and the Winkler elastic foundation parameter on the fundamental frequencies of the nanobeam is investigated and tabulated. Also, in the present study, Poisson's ratio is taken as constant. Nanobeam resting on the Winkler foundation which is simply supported is analyzed to illustrate the size effects on the free vibration. Numerical results for the simply supported nanobeam indicate that the first fundamental frequency calculated by the presented model is higher than the classical one. Moreover, it is obtained that the size influence is more substantial for higher vibration modes. The results indicate that the significant importance of the size influences the analysis of nanobeams. The vibration of nanobeam exhibits a hardening spring behavior, and the newly developed models are the beams stiffer than according to the classical beam theory. Modified couple stress theory tends to be more helpful in describing the size-dependent mechanical properties of nanoelectromechanical systems (NEMS).
\end{abstract}

Keywords: nanobeam; modified couple-stress theory; perturbation methods; Winkler foundation

\section{INTRODUCTION}

In recent years, research on nanostructures, which are used as a fundamental part of a large number of micro and nanoelectromechanical systems (MEMS and NEMS), has received great interest from researchers. Standard MEMS and NEMS consist of conventional silicon-based materials, metal thin beams, functional graded materials, or polymers. The dynamic and mechanical behavior of nanostructures has been particularly interesting for scientists. The method of the development of non-classical continuity theory is substantial to model the nano- and micro-structures in the aspects of dynamic and static properties, because it is time-consuming and difficult to conduct experiments at the nanoscale. The inclusion of material length parameters in the equations of motion for defining size effect is possible with non-classical continuum theories for analyzing nanostructures [1].

Nowadays, modified couple stress theory (MCST), which is a size-dependent continuum theory, has been an attractive research subject for scientists and engineers. Mechanical properties of nanostructures in terms of linear and nonlinear are discussed by introducing the size effect in motion equations. Park and Gao [2] initially applied the modified couple stress theory into the study to examine the microcantilever Euler-Bernoulli beam under the influence of a point load in static deformation.

Recently, couple stress effect is being used by scientists to investigate the size influence of nanostructures. In the literature survey, mechanical characteristics of nanobeams are investigated using various beam models on the basis of Euler-Bernoulli, Timoshenko and Reddy beam theories to analyze the dimensional effect. Double-walled boron nitride nanotubes (DWBNNTs) conveying fluid embedded in a viscoelastic foundation is modeled to investigate the geometric nonlinearity effect, the size effect, transverse shear deformation effect, and rotary inertia effect [3]. In their work, they have considered the viscoelastic medium spring and damping constants, the small-scale coefficient and instability behaviors of DWBNNT and fluid velocity on vibration. The results of their studies indicated that the damping constant has a substantial influence on the critical flow velocity and resonant frequencies. Double-walled carbon nanotubes (DWCNTs) that convey fluid are examined with the aid of the modified couple stress theory to analyze the vibration and instability characteristics. The results of their studies showed that the effect of length scale parameter performs a significant role in the fluid conveying DWCNNTs frequency and critical flow velocity. Şimşek and Reddy $[5,6]$ performed a different beam theory on the base of the modified couple stress theory to analyze the free vibration and buckling of functionally graded microbeams. Simsek [7] focused on the static bending of microbeams in large amplitude and free vibration of microbeams on the nonlinear elastic medium by employing Euler-Bernoulli beam theory and modified couple stress theory. His results indicated that the increment of the dimensionless scale parameter decreases the nonlinear frequency ratio. Moreover, nonlinear foundation parameter has an opposite influence on the nonlinear frequency related to Winkler and Pasternak parameters. Barooti and Ghadiri [8] studied the critical speed and free vibration analysis of spinning 3D SWCNTs rested on an elastic medium considering the modified couple stress theory. They have concluded that material length scale parameter and the angular velocity have an important effect on the rotating SWCNTs fundamental frequencies.

Eringen [9] has developed a size-dependent theory titled as a nonlocal elasticity theory. According to this theory, the stress state at a given reference point is a function of the strain field at every point in the body.

Hence, the nonlocal continuum mechanics theory includes information about the internal length scale and the long-range forces between atoms are inserted into the structural equations just as the parameter of material length 
scale. Peddieson et al. [10] have been first to apply the theory of nonlocal Euler-Bernoulli beam to nano- and microstructures. In that study, Peddieson et al. [10] studied the cantilever beam by applying the newly developed theory called a nonlocal Euler-Bernoulli beam. For nanomaterials, the theory of non-local elasticity was suggested to reveal the scale effects. Eringen's nonlocal differential constitutive relation has been applied by Soltani et al. [11] to analyze the nonlinear vibration characteristics of the fluid-conveying CNTs. Damping and elastic medium effect on critical flow velocity and resonant frequency have been discussed by Soltani et a. [11]. With the help of the nonlocal elasticity model, effect of viscoelastic foundation on the cantilever carbon nanotubes to obtain the structural stability [12], nanobeam rested on an elastic medium to analyze the vibration characteristics [13] and nanobeam rested on Pasternak type elastic medium [14], fluid-conveying nonuniform CNTs embedded in viscoelastic medium to analyze the vibration responses [15], nanotube embedded in viscous matrix to analyze the nonlinear free vibrations [16], DWCNTs that convey fluid embedded in viscoelastic medium to analyze the thermal-mechanical vibration and instabilities [17] have been investigated by researchers.

Theory of Eringen's nonlocal elasticity is one of the best and well-known theories by all the researchers who studied the nonlocal continuum mechanics. That theory includes the effect of small scale with enough precision in micro/nanoscale equipment. In addition to this theory, the modified couple stress theory is also the valuable theory. The researches indicated that the modified couple stress theory is compatible with the experimental results of Eringen's nonlocal elasticity and classical theories [9]. Therefore, this is the reason why the theory of modified couple stress theory has been introduced in governing equations in the present study.

All of the useful studies mentioned in the literature review employed the theory of Eringen's nonlocal elasticity to microbeam and nanobeam. Moreover, most of the studies examine the vibration characteristics of nanobeam resting on an elastic medium on the basis of Eringen's nonlocal elasticity theory. In the present study, nanobeam resting on an elastic medium is examined to analyze the vibration characteristics using the modified couple stress theory. The length scale parameter effect and linear foundation stiffness coefficients on the linear frequency are analyzed. In addition, considerable numerical data is presented in tabulated form for various values of the parameters; hence in the future, these results may be used as a reference.

\section{SIZE DEPENDENT NANOBEAM RESTING ON AN ELASTIC FOUNDATION EQUATIONS \\ 2.1 The Modified Couple Stress Theory}

The scope of modified couple stress theory was firstly presented by Yang et al. [18]. Based on this theory, the strain energy is a function of both the rotation gradient tensor and strain tensor. Moreover, it consists of two Láme parameters and one length scale parameter. When we look at this theory, the strain energy follows as:

$$
U=\frac{1}{2} \iiint_{V}\left(\sigma_{i j} \varepsilon_{i j}+m_{i j} \chi_{i j}\right) \mathrm{d} V
$$

where $\sigma_{i j}$ is the stress tensor, $\varepsilon_{i j}$ is the strain tensor, $m_{i j}$ is the higher order stress tensor and $\chi_{i j}$ is symmetric rotation gradient tensor. These tensors are given as

$$
\begin{aligned}
\sigma_{i j} & =\lambda \varepsilon_{k k} \delta_{i j}+2 \mu \varepsilon_{i j} \\
\varepsilon_{i j} & =\frac{1}{2}\left(u_{i, j}+u_{j, i}\right) \\
m_{i j} & =2 \mu l^{2} \chi_{i j} \\
\chi_{i j} & =\frac{1}{2}\left(\theta_{i, j}+\theta_{j, i}\right)
\end{aligned}
$$

where $u_{i}$ is the displacement vector and $\delta_{i j}$ is the Kronecker delta. $l$ is the material length scale parameter and $\theta_{i}$ is the rotation vector that can be given as

$\theta_{i}=\frac{1}{2} e_{i j k} u_{k, j}$

where $e_{i j k}$ is the permutation symbol. $\lambda$ and $\mu$ are the Láme's constants that are given as

$$
\lambda=\frac{E v}{(1+v)(1-2 v)}, \mu=\frac{E}{2(1+v)}
$$

where $v, \mu$ and $E$ are Poisson's ratio, shear modulus and Young's modulus, respectively.

\subsection{Governing Equations and Boundary Conditions}

Hamilton's principle and the modified couple stress theory that allow to derive the equations of motion and boundary conditions to construct the governing equations are derived for nanobeam, for the Euler-Bernoulli beam model, resting on the elastic medium. Fig. 1 schematically represents a simply supported nanobeam.

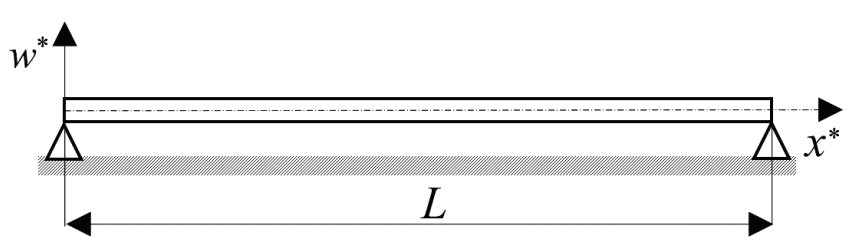

Figure 1 Simply supported beam

For the system shown in Fig. 1, w* is the beam transverse displacement between supports and $L$ is the length of the beam. Lagrange equation is given in Eq. (8), $\rho A$ is the mass per unit length, $t^{*}$ is the time, $N^{*}$ is the axial force, $E I$ is flexural rigidity and $E A$ is longitudinal rigidity. 


$$
\begin{aligned}
& £=\frac{1}{2} \int_{0}^{L} \rho A\left(\frac{\partial w^{*}}{\partial t^{*}}\right)^{2} \mathrm{~d} x^{*}-\frac{1}{2} \int_{0}^{L} E I\left(\frac{\partial^{2} w^{*}}{\partial x^{* 2}}\right)^{2} \mathrm{~d} x^{*}- \\
& -\frac{1}{2} \frac{E}{2(1+v)} A l^{2} \int_{0}^{L}\left(\frac{\partial^{2} w^{*}}{\partial x^{* 2}}\right)^{2} \mathrm{~d} x^{*}- \\
& -\frac{1}{2} \int_{0}^{L} N^{*}\left(\frac{\partial w^{*}}{\partial x^{*}}\right)^{2} \mathrm{~d} x^{*}-\frac{1}{2} \int_{0}^{L} k w^{* 2} \mathrm{~d} x^{*}
\end{aligned}
$$

The governing equation of motion of nanobeam considering the dimensional form is acquired with the help of Hamilton's principle and by implementing the necessary algebra:

$$
\begin{aligned}
& \left(E I+\frac{E}{2(1+v)} A l^{2}\right) \frac{\partial^{4} w^{*}}{\partial x^{* 4}}+\rho A \frac{\partial^{2} w^{*}}{\partial t^{* 2}}+k w^{*}= \\
& =\frac{E A}{2 L}\left[\int_{0}^{L}\left(\frac{\partial w^{*}}{\partial x^{*}}\right)^{2} \mathrm{~d} x^{*}\right] \frac{\partial^{2} w^{*}}{\partial x^{* 2}}
\end{aligned}
$$

The term $\mu A l^{2}\left(\partial^{4} w^{*} / \partial x^{* 4}\right)$ comes from modified couple stress theory. Nanobeam nanostructure has been introduced into the equation by considering the material length scale parameter $l$. The corresponding boundary conditions at the ends of the beam $\left(x^{*}=0\right.$ and $\left.x^{*}=L\right)$ are given as:

Simple-Simple Case:

$$
\begin{aligned}
& w^{*}(0)=0, \quad w^{*}(L)=0 \\
& w^{* \prime \prime}(0)=0, \quad w^{* \prime \prime}(L)=0
\end{aligned}
$$

the dimensionless form by considering the Eqs. (9) and (10) can be described as:

$$
\begin{aligned}
& \bar{x}=\frac{x}{L}, \bar{w}=\frac{w}{L}, \bar{t}=\frac{t}{L^{2}} \sqrt{\frac{E I}{\rho A}}, \\
& \xi=\frac{h}{l}, \eta=\frac{6}{(1+v) \xi^{2}}, \kappa=\frac{k L^{4}}{E I} \\
& (1+\eta) \frac{\partial^{4} \bar{w}}{\partial \bar{x}^{4}}+\frac{\partial^{2} \bar{w}}{\partial \bar{t}^{2}}+\kappa \bar{w}=\frac{1}{2}\left[\int_{0}^{1}\left(\frac{\partial \bar{w}}{\partial \bar{x}}\right)^{2} \mathrm{~d} \bar{x}\right] \frac{\partial^{2} \bar{w}}{\partial \bar{x}^{2}}
\end{aligned}
$$

The dimensionless form of boundary conditions at the end of beam is as follows:

Simple-Simple Case:

$$
\bar{w}(0)=0, \quad \bar{w}(1)=0
$$$$
\bar{w}^{\prime \prime}(0)=0, \quad \bar{w}^{\prime \prime}(1)=0
$$

\section{SOLUTIONS USING A PERTURBATION TECHNIQUE}

In this part of the study, an analytical solution will be approximately obtained with the help of the multiple scale methods having an important perturbation technique. According to the following expansion,

$\bar{w}(x, t ; \varepsilon)=\bar{w}_{0}\left(x, T_{0} ; T_{1}\right)+\varepsilon \bar{w}_{1}\left(x, T_{0} ; T_{1}\right)$

where $T_{0}=T, T_{1}=\varepsilon T$ and $\varepsilon<<1$ [19-20]. $\bar{\mu}=\varepsilon \mu$, $\bar{F}=\varepsilon \sqrt{\varepsilon} F$ the transformation is implemented for the forcing and damping terms on the basis of the multiple scale method [19, 20], respectively. Introducing,

$$
\frac{\partial}{\partial t}=D_{0}+\varepsilon D_{1}, \frac{\partial^{2}}{\partial t^{2}}=D_{0}^{2}+2 \varepsilon D_{0} D_{1}
$$

where $D_{n}=\partial / \partial T_{n}$. After making the necessary expansion, the different order of motion equations and boundary conditions are given in the following form:

Order (1):

$(1+\eta) \bar{w}_{0}^{i v}+D_{0}^{2} \bar{w}_{0}-v_{p}^{2} \overline{\mathrm{w}}_{0}=0$

Order $(\varepsilon)$ :

$(1+\eta) \bar{w}_{1}^{i v}+D_{0}^{2} \bar{w}_{1}-v_{p}^{2} \bar{w}_{1}=-2 D_{0} D_{1} \bar{w}_{0}+$

$+\frac{1}{2}\left[\int_{0}^{1} \bar{w}_{0}^{\prime \prime 2} \mathrm{~d} x\right] \bar{w}_{0}^{\prime \prime}+F \cos \Omega t-2 \mu D_{0} \bar{w}_{0}$

Fundamental frequency which possesses first order of expansion can be obtained.

\subsection{Linear Problem}

Linear problem is frequently represented by the first order of equation given in Eq. (16). The problem solution is given in the complex form as:

$\bar{w}_{0}\left(x, T_{0}, T_{1}\right)=A\left(T_{1}\right) \mathrm{e}^{i \omega \mathrm{T}_{0}} Y(x)+\bar{A}\left(T_{1}\right) \mathrm{e}^{-i \omega \mathrm{T}_{0}} \bar{Y}(x)$

$A$ is the complex amplitude. Substituting Eq. (18) into Eq. (17), the following can be obtained:

$(1+\eta) Y^{i v}(x)-\frac{\omega^{2}}{\alpha} Y(x)=0$

The solution of $Y(x)$ can be obtained as follows:

$Y(x)=c_{1} \mathrm{e}^{i \beta_{1} x}+c_{2} \mathrm{e}^{i \beta_{2} x}+c_{3} \mathrm{e}^{i \beta_{3} x}+c_{4} \mathrm{e}^{i \beta_{4} x}$

Since the boundary conditions are considered, the frequency equations are obtained. 


\section{NUMERICAL RESULTS}

All the obtained fundamental frequency values in this study consider the modified couple stress theory and EulerBernoulli beam theory. Influence of the dimensionless material length scale parameter $h / l$ is indicated by the $\zeta$ symbol. Since it is working in dimensionless form, the dimensionless Winkler spring constant $(\kappa)$ and dimensionless material length scale parameter $(\zeta)$ are sufficient to calculate the results. In Tab. 1, the fundamental frequencies with different length scale parameter, the Winkler spring constant and simply supported boundary are given. In this table, Poisson's ratio is taken as $v=0.38$, and the dimensionless material length scale parameter with $h / l=1,2,3,4$ and 5 and the dimensionless Winkler foundation parameter with $\kappa=$ 0,100 and 500 , respectively. It is obvious from the table that the natural frequencies increase once the dimensionless Winkler foundation parameter $(\kappa)$ increases. This is because increasing the Winkler foundation parameters increases the stiffness of the beam. It is well known that the fundamental frequencies of a system generally increase the increment of the foundation stiffness. On the other hand, Tab. 1 reveals that increment in the scale parameter causes the decrease in the fundamental frequencies. It is known that the influence of the small scale has an effect on the nanotube mechanical behaviors.

Table 1 The first five frequencies for different $\kappa$ and $\xi$ values and $v=0.38$ for Simple-Simple support condition

\begin{tabular}{|c|c|c|c|c|c|}
\hline \multicolumn{6}{|c|}{$\kappa=0$} \\
\hline$\xi$ & 1 & 2 & 3 & 4 & 5 \\
\hline$\omega_{1}$ & 22.8238 & 14.2579 & 12.0194 & 11.1301 & 10.6934 \\
\hline$\omega_{2}$ & 91.2953 & 57.0317 & 48.0777 & 44.5204 & 42.7738 \\
\hline$\omega_{3}$ & 205.414 & 128.321 & 108.175 & 100.171 & 96.241 \\
\hline$\omega_{4}$ & 365.181 & 228.127 & 192.311 & 178.081 & 171.095 \\
\hline$\omega_{5}$ & 570.596 & 356.448 & 300.486 & 278.252 & 267.336 \\
\hline \multicolumn{5}{|c|}{$\kappa=100$} \\
\hline$\xi$ & 1 & 2 & 3 & 4 & 5 \\
\hline$\omega_{1}$ & 24.9184 & 17.4152 & 15.6354 & 14.9626 & 14.6407 \\
\hline$\omega_{2}$ & 91.8413 & 57.9018 & 49.1067 & 45.6296 & 43.9272 \\
\hline$\omega_{3}$ & 205.658 & 128.71 & 108.636 & 100.669 & 96.7592 \\
\hline$\omega_{4}$ & 365.318 & 228.346 & 192.571 & 178.362 & 171.387 \\
\hline$\omega_{5}$ & 570.683 & 356.588 & 300.652 & 278.432 & 267.523 \\
\hline \multicolumn{7}{|c|}{$\kappa=500$} \\
\hline$\xi$ & 1 & 2 & 3 & 4 & 5 \\
\hline$\omega_{1}$ & 31.9519 & 26.5196 & 25.3863 & 24.9776 & 24.7861 \\
\hline$\omega_{2}$ & 93.9938 & 61.2586 & 53.0233 & 49.8203 & 48.2659 \\
\hline$\omega_{3}$ & 206.628 & 130.255 & 110.462 & 102.636 & 98.8045 \\
\hline$\omega_{4}$ & 365.865 & 229.22 & 193.606 & 179.48 & 172.55 \\
\hline$\omega_{5}$ & 571.034 & 357.149 & 301.317 & 279.149 & 268.27 \\
\hline
\end{tabular}

\section{CONCLUDING REMARKS}

In this study, modified couple stress theory is proposed for the nanobeam which is on an elastic foundation. The simply supported nanobeam has been compared with the Euler-Bernoulli beam type as a benchmark for beam analysis study. Results for natural frequencies are obtained with multiple scale method. The influences of non-dimensional material length scale parameter $(\xi)$, non-dimensional Winkler foundation parameter $(\kappa)$ and simply supported boundary condition are tabulated. It was found that:

- Non-dimensional material length scale parameter $(\xi)$ effect plays a substantial role in the fundamental frequency of the nanobeam. The natural frequency is declined with the increment of dimensionless material length scale parameter. This effect leads to softening the nanobeam.

- The non-dimensional Winkler spring constant $(\kappa)$ is increasing with the increase of fundamental frequency of the nanobeam. This effect increases the system stiffness.

- All values of the numerical results show that the fundamental frequency of the nanobeam decreases with increment of the non-dimensional material length scale parameters and increases with increment of nondimensional Winkler foundation parameters.

\section{REFERENCES}

[1] Iijima, S. (1991). Helical microtubules of graphitic carbon. Nature, 354(6348), 56. https://doi.org/10.1038/354056a0

[2] Park, S. K. \& Gao, X. L. (2006). Bernoulli-Euler beam model based on a modified couple stress theory. Journal of Micromechanics and Microengineering; 16, 2355-2359. https://doi.org/10.1088/0960-1317/16/11/015

[3] Ghorbanpour, A. A. et al. (2013). Nonlinear vibration and instability of fluid-conveying DWBNNT embedded in a viscoPasternak medium using modified couple stress theory. Journal of Mechanical Science and Technology, 27(9), 26452658. https://doi.org/10.1007/s12206-013-0709-3

[4] Ke, L. L. \& Wang, Y. S. (2011). Flow-induced vibration and instability of embedded double-walled carbon nanotubes based on a modified couple stress theory. Physica E: Lowdimensional Systems and Nanostructures, 43(5), 1031-1039. https://doi.org/10.1016/j.physe.2010.12.010

[5] Şimşek, M. \& Reddy, J. N. (2013). Bending and vibration of functionally graded microbeams using a new higher order beam theory and the modified couple stress theory. International Journal of Engineering Science, 64, 3753. https://doi.org/10.1016/j.jiengsci.2012.12.002

[6] Şimşek, M. \& Reddy, J. N. (2013). A unified higher order beam theory for buckling of a functionally graded microbeam embedded in elastic medium using modified couple stress theory. Composite Structures, 101, 47-58. https://doi.org/10.1016/j.compstruct.2013.01.017

[7] Şimșek, M. (2014). Nonlinear static and free vibration analysis of microbeams based on the nonlinear elastic foundation using modified couple stress theory and He's variational method. Composite Structures, 112, 264-272. https://doi.org/10.1016/j.compstruct.2014.02.010

[8] Barooti, M. M., Safarpour, H., \& Ghadiri, M. (2017). Critical speed and free vibration analysis of spinning 3D single-walled carbon nanotubes resting on elastic foundations. The European Physical Journal Plus, 132(1), 6. https://doi.org/10.1140/epjp/i2017-11275-5

[9] Eringen, A. C. (1983). On differential equations of nonlocal elasticity and solutions of screw dislocation and surface waves. Journal of applied physics, 54(9), 4703-4710. https://doi.org/10.1063/1.332803

[10] Peddieson, J., Buchanan, G. R., \& McNitt, R. P. (2003). Application of nonlocal continuum models to nanotechnology. International Journal of Engineering Science, 41(3-5), 305312. https://doi.org/10.1016/S0020-7225(02)00210-0 
[11] Soltani, P., Taherian, M. M., \& Farshidianfar, A. (2010). Vibration and instability of a viscous-fluid-conveying singlewalled carbon nanotube embedded in a visco-elastic medium. Journal of Physics D: Applied Physics, 43(42), 425401. https://doi.org/10.1088/0022-3727/43/42/425401

[12] Kazemi-Lari, M. A., Fazelzadeh, S. A., \& Ghavanloo, E. (2012). Non-conservative instability of cantilever carbon nanotubes resting on viscoelastic foundation. Physica E: Lowdimensional Systems and Nanostructures, 44(7-8), 1623-1630. https://doi.org/10.1016/j.physe.2012.04.007

[13] Togun, N. (2016). Nonlocal beam theory for nonlinear vibrations of a nanobeam resting on elastic foundation. Boundary Value Problems, 2016(1), 57. https://doi.org/10.1186/s13661-016-0561-3

[14] Togun, N. \& Bağdatlı, S. M. (2016). Nonlinear vibration of a nanobeam on a Pasternak elastic foundation based on non-local Euler-Bernoulli beam theory. Mathematical and Computational Applications, 21(1), 3. https://doi.org/10.3390/mca21010003

[15] Rafiei, M., Mohebpour, S. R., \& Daneshmand, F. (2012). Small-scale effect on the vibration of non-uniform carbon nanotubes conveying fluid and embedded in viscoelastic medium. Physica E: Low-Dimensional Systems and Nanostructures, 44(7-8), 1372-1379. https://doi.org/10.1016/j.physe.2012.02.021

[16] Wang, Y. Z. \& Li, F. M. (2014). Nonlinear free vibration of nanotube with small scale effects embedded in viscous matrix. Mechanics Research Communications, 60, 45-51. https://doi.org/10.1016/j.mechrescom.2014.06.002

[17] Zhen, Y. X., Fang, B., \& Tang, Y. (2011). Thermal-mechanical vibration and instability analysis of fluid-conveying double walled carbon nanotubes embedded in visco-elastic medium. Physica E: Low-dimensional Systems and Nanostructures, 44(2), 379-385. https://doi.org/10.1016/j.physe.2011.09.004

[18] Yang, F. A. C. M., Chong, A. C. M., Lam, D. C. C., \& Tong, P. (2002). Couple stress based strain gradient theory for elasticity. International Journal of Solids and Structures, 39(10), 2731-2743. https://doi.org/10.1016/S0020-7683(02)00152-X

[19] Nayfeh, A. H. \& Mook, D. T. (2008). Nonlinear oscillations. John Wiley \& Sons.

[20] Nayfeh, A. H. (2011). Introduction to perturbation techniques. John Wiley \& Sons.

\section{Authors' contacts:}

Necla TOĞUN, PhD, Associate Professor

Vocational School of Technical Sciences in Gaziantep,

Gaziantep University, 27310, Gaziantep, Turkey

Tel.: +903423171791,

E-mail: nkara@gantep.edu.tr

Süleyman M. BAĞDATLI, PhD, Associate Professor

Faculty of Engineering, Department of Mechanical Engineering,

Manisa Celal Bayar University, 45140, Yunusemre, Manisa, Turkey

Tel.: +902362012368/Fax: +902012020,

E-mail: murat.bagdatli@cbu.edu.tr 